01.3;01.5;02.3

\title{
Мобильные диссипативные бризеры в цепочке нелинейных осцилляторов
}

\author{
() К.С. Сергеев, Е.М. Елизаров, А.П. Четвериков
}

Саратовский национальный исследовательский государственный университет им. Н.Г. Чернышевского, Саратов, Россия E-mail: kssergeev@mail.ru

Поступило в Редакцию 10 июня 2020 г.

В окончательной редакции 10 июня 2020 г.

Принято к публикации 25 июля 2020 г.

\begin{abstract}
В численном эксперименте обнаружено, что в цепочке осцилляторов Рэлея с нелинейной связью между ними возможно существование как минимум трех локализованных стационарных мод бризерного типа: немобильные, „медленные“ и „быстрые“ диссипативные бризеры. Динамика синхронных движений элементов цепочки зависит от соотношения характерных временны́х масштабов системы, связанных с частотой осцилляторов и жесткостью связи между ними, и является мультистабильной.
\end{abstract}

Ключевые слова: цепочки осцилляторов, активные частицы, нелинейные системы, нелинейная связь, диссипативные дискретные бризеры.

DOI: 10.21883/PJTF.2020.21.50191.18418

Ансамбли элементов различной природы, взаимодействующих посредством связи того или иного рода, демонстрируют много типов синхронного коллективного поведения. Особый интерес представляет динамика упорядоченных систем взаимодействующих частиц, которые могут быть как консервативными [1,2], так и активными [3-5], и ансамблей связанных осцилляторов [6-9]. Варьированием параметров элементов и связей модели, описывающей упорядоченный ансамбль осцилляторов, можно получать в асимптотике известные модели цепочки консервативных частиц (Тоды, Морзе, Леннарда-Джонса, Ферми-Паста-Улама) и цепочки активных частиц (например, Рэлея-Морзе [10,11]), а также цепочки консервативных осцилляторов [7] и цепочки активных осцилляторов $[6,8]$. В настоящей работе исследуется поведение цепочек активных частиц и активных осцилляторов, которые переходят друг в друга при стремлении частоты осцилляторов к нулю.

Рассматривается динамика цепочки активных частиц с трением Рэлея [3,4]:

$$
\gamma(v)=-\mu\left(1-\frac{v^{2}}{v_{0}^{2}}\right),
$$

связь между которыми обеспечивается силами, соответствующими потенциалу Морзе $U(r)=D\left(e^{-2 b r}-2 e^{-b r}\right)$, и сопоставляется с динамикой аналогичной цепочки осцилляторов Рэлея с частотой $\tilde{\omega}_{R}$. Динамика каждого элемента рассматриваемой цепочки точечных масс (элементов) задается безразмерным уравнением

$$
\begin{aligned}
\ddot{q}_{i}-\mu\left(1-\frac{\dot{q}_{i}^{2}}{v^{2}}\right) \dot{q}_{i}^{2}+\omega_{r}^{2} q_{i}=\left(1-e^{q_{i}-q_{i+1}}\right) e^{q_{i}-q_{i+1}} \\
-\left(1-e^{q_{i-1}-q_{i}}\right) e^{q_{i-1}-q_{i}} .
\end{aligned}
$$

Здесь величина $q_{i}=b\left(x_{i}-i \sigma\right)$ - безразмерное отклонение $i$-го элемента от его равновесного положения $x_{i 0}=i \sigma$ в невозмущенной цепочке, $\sigma$ опреде- ляет равновесное расстояние между соседними элементами, $\tau=\tilde{\omega}_{M} t$ определяет безразмерное время, где $\tilde{\omega}_{M}=\sqrt{2 D b^{2} / m}-$ частота линейных колебаний частиц цепочки за счет связи вблизи положений равновесия, $\omega_{r}$ - частота линейных колебаний осцилляторов в единицах $\tilde{\omega}_{M}$. Безразмерные параметры $v_{0}=\tilde{v}_{0} b / \tilde{\omega}_{M}$ и $\mu=\tilde{\mu} / m \tilde{\omega}_{M}-$ стационарная скорость частицы и линейный коэффициент отрицательного трения Рэлея соответственно, $m$ - масса частицы, $D$ и $b-$ глубина потенциальной ямы и коэффициент жесткости потенциала Морзе. Частоты $\omega_{r}$ и $\omega_{M}\left(\omega_{M}=1\right.$ в единицах $\left.\tilde{\omega}_{M}\right)$ соответствуют двум характерным временны́м масштабам цепочки осцилляторов, определяемых онсайтовыми и интер-сайтовыми колебаниями соответственно. При $\omega_{r}=0$ цепочка осцилляторов трансформируется в цепочку активных частиц. Анализ динамики цепочки производится на основе результатов численного моделирования цепочки 48 осцилляторов/частиц (1) с периодическими граничными условиями, выполненного методом Рунге-Кутты четвертого порядка с шагом интегрирования $10^{-3}$.

Как было показано в [4,5,10-12], вдоль цепочки активных частиц могут распространяться локализованные волны - диссипативные солитоны - в виде максимумов скорости и плотности частиц. Для возбуждения солитонов можно использовать начальные условия, определяемые как случайными возмущениями скоростей и координат частиц, так и специальным выбором возмущений скоростей и координат одной или нескольких смежных частиц. Эти же волны играют роль асимптотических для цепочки осцилляторов при стремлении их частоты собственных колебаний к нулю. При увеличении частоты $\omega_{r}$ от нуля возбуждение диссипативных солитонов также возможно, пока выполняется условие $\omega_{r} \ll \omega_{m}$, однако время жизни солитонов в таких цепочках ограничено периодом осцилляций Рэлея (рис. 1,a). В момент смены 


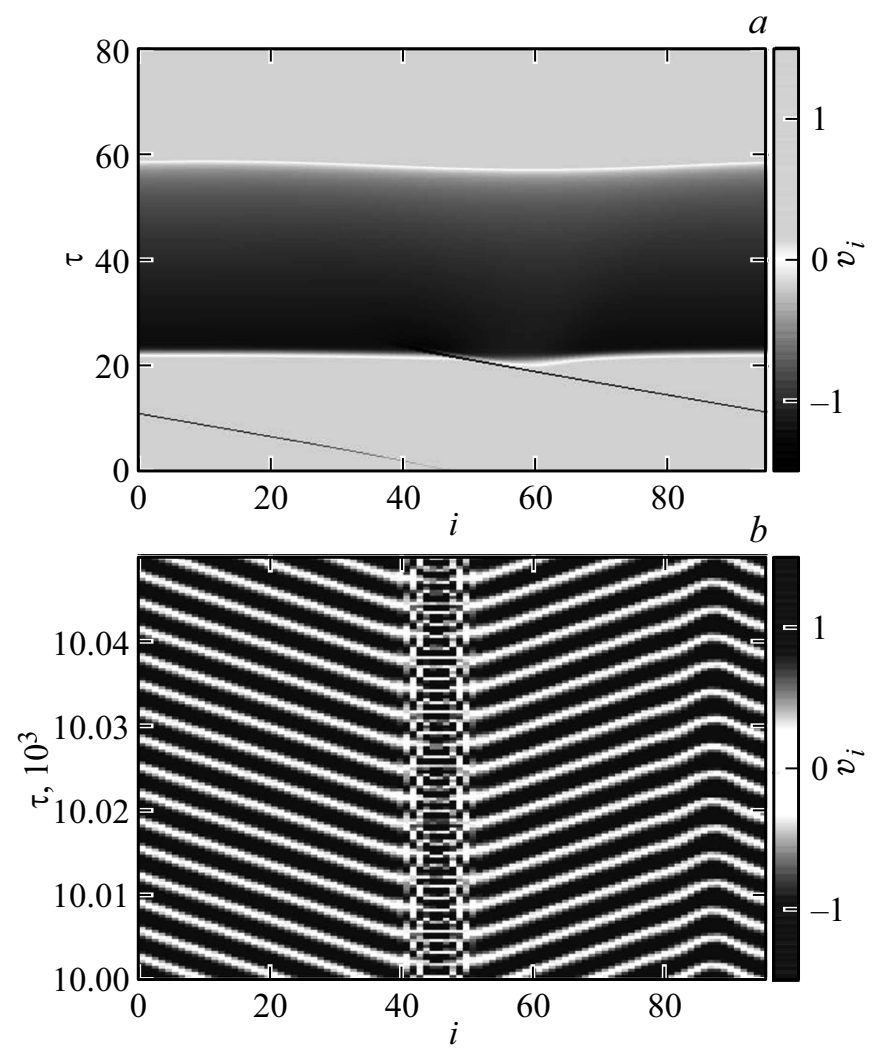

Рис. 1. Эволюция распределения скорости частиц при возбуждении солитона и немобильного диссипативного дискретного бризера. $a-$ солитон, параметры цепочки: $b \sigma=2, \omega_{r}^{2}=0.1$, $\mu=0.7 ; b-$ диссипативный дискретный бризер, параметры цепочки: $b \sigma=2.02, \omega_{r}^{2}=11, \mu=0.35$.

знака скорости $\dot{q}$ солитоны разрушаются, а возмущения цепочки со временем переходят в пространственно однородный режим [12] (в терминах, применяемых при описании цепочек частиц, такой режим называют режимом трансляции).

Помимо тривиальных решений (полной синфазной и противофазной синхронизации и режимов бегущих волн) в цепочке обнаружен особый вид локализованных возбуждений, которые по форме похожи на дискретные бризеры $[1,2]$ в консервативных решетках.

В качестве иллюстрации на рис. $1, b$ приведена пространственно-временна́я диаграмма такой моды при $\omega_{r}=3.3$, которую уместно обозначить термином „диссипативный дискретный бризер“ (ДБ). Легко различим кластер частиц, динамика которых качественно отличается от поведения частиц остальной части ансамбля, в которой распространяется пара бегущих волн. Динамика осцилляций внутри этого кластера соответствует колебаниям за счет сил потенциала Морзе, тогда как характеристики бегущих волн определяются свойствами осцилляторов Рэлея и граничными условиями. Соседние элементы, составляющие бризер, колеблются противофазно и квазипериодически, что подтверждается расчетами автокорреляционной функции и спектров (здесь не приводятся).
Число элементов, формирующих диссипативный ДБ, зависит от начальных условий, однако монотонной зависимости размера кластера от количества и интенсивности изначально возмущаемых осцилляторов не обнаружено. Время жизни обнаруженных мод потенциально бесконечно; по крайней мере, после завершения относительно коротких переходных процессов при симуляции в течение времени порядка $10^{6} \omega_{M}$ никаких изменений в структуре диссипативных ДБ обнаружено не было. В частности, бризер на рис. $1, b$ остается локализованным на тех же осцилляторах, а форма колебаний и их спектры не изменяются по прошествии обозначенного выше времени симуляции.

Однако помимо описанных „немобильных“ диссипативных ДБ выбором параметров и начальных условий можно возбудить бризеры, перемещающиеся по цепочке с некоторой скоростью. В качестве примера на рис. $2, a, b$ приведены пространственно-временны́е диаграммы для двух мобильных диссипативных ДБ, полученные в цепочках с разными наборами параметров при одних и тех начальных условиях, которые задавались в следующем виде: скорости $\dot{q}_{i}$ всех элементов равны нулю, кроме трех, для которых $\dot{q}_{24}=0.5, \dot{q}_{28}=-1, \dot{q}_{31}=1$ (смещения $q_{i}=0 \forall i$ ). При расчетах с заданными началь-
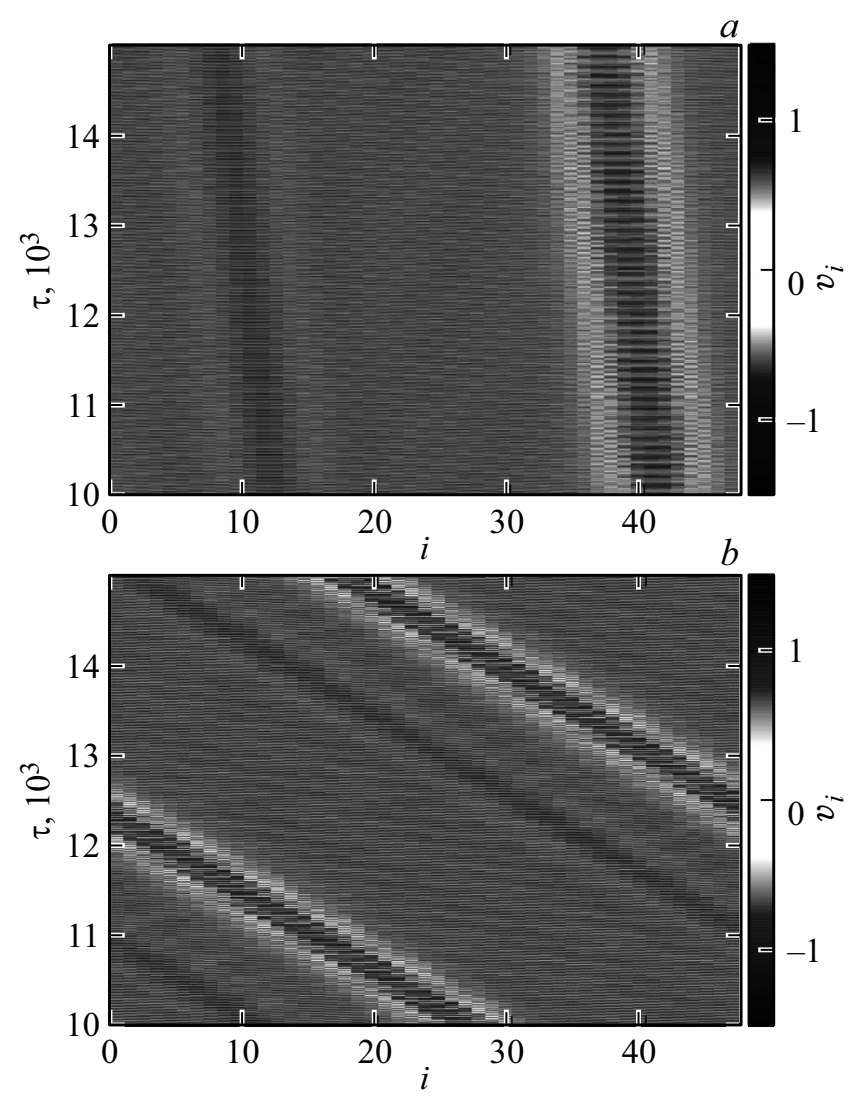

Рис. 2. Эволюция распределения скоростей частиц при распространении диссипативных дискретных бризеров, движущихся с различными скоростями. $a-$ скорость бризера 0.003 , параметры цепочки: $b \sigma=2, \omega_{r}^{2}=9, \mu=0.35 ; b-$ скорость бризера 0.04, параметры цепочки: $b \sigma=2.06, \omega_{r}^{2}=12$, $\mu=0.35$. 


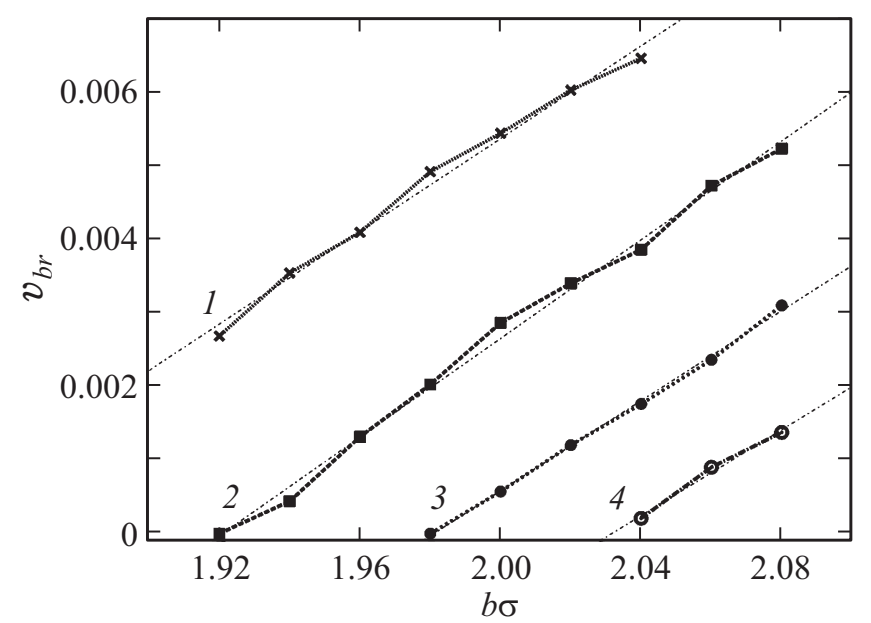

Рис. 3. Семейство зависимостей скорости $v_{b r}$ мобильного бризера от параметра $b \sigma$ при различных значениях $\omega_{r}^{2} . \omega_{r}^{2}=8(1)$, 9 (2), 10 (3) и 11 (4). Полученные из численного эксперимента зависимости имеют линейный характер и аппроксимируются прямыми с одинаковым наклоном (показаны тонкими штрихпунктирными линиями). $\mu=0.35$.

ными условиями наблюдается относительно короткий (порядка $10^{3} \omega_{M}$ ) переходный процесс, в течение которого могут изменяться размер и скорость бризера. По завершении всех переходных процессов бризер становится стационарным и в течение длительного времени движется с постоянной скоростью и не деформируется.

Для цепочки определенной длины при заданной форме начальных условий скорость сформировавшегося бризера зависит от значений остальных параметров. Наибольшее влияние на скорость оказывают жесткость потенциальных сил $b \sigma$ и частота осцилляторов Рэлея $\omega_{r}$ (рис. 3). Из рис. 3 видно, что увеличение частоты осциллятора $\omega_{r}$ ведет к снижению скорости бризера, a с ростом $b \sigma$ скорость растет асимптотически линейно. Более того, наклон всех линейных аппроксимаций (тонкие штрихпунктирные линии) примерно одинаков и составляет 0.03. Диапазон изменения параметров на представленном рисунке для каждой кривой соответствует области существования данного мобильного бризера. Внутри этой области при варьировании параметров скорость бризера меняется плавно и монотонно, тогда как при выходе из нее происходит резкий скачок скорости бризера (а иногда и его формы, т.е. числа осцилляторов, вовлеченных в бризер). Так, например, в результате изменения значения $\omega_{r}^{2}$ с 11 на 12 при $b \sigma=2.06$ вместо бризера, представленного на рис. 2, $a$, движущегося со скоростью 0.003 (условно „медленный“ бризер), формируется бризер, показанный на рис. 2, $b$, движущийся со скоростью 0.04 (условно „быстрый“). При варьировании значений параметров в широких пределах с сохраненем формы начальных условий в исследуемой цепочке были обнаружены только стационарные, „быстрые“ и „медленные“ мобильные бризеры.

Таким образом, исследуемая цепочка осцилляторов является мультистабильной. Ее стационарные моды - диссипативные дискретные бризеры - являются аттракторами с очень узкими бассейнами притяжения, причем взаимное расположение их бассейнов притяжения имеет, по-видимому, сложную структуру (малые изменения формы начальных условий приводят к выходу цепочки на другой аттрактор). Сами диссипативные бризеры могут быть как мобильными, движущимися по цепочке с периодическими граничными условиями с постоянной скоростью, так и немобильными, локализованными на одном и том же кластере осцилляторов. При малых изменениях параметров цепочки монотонно меняется скорость бризера, а если изменения параметров значительны, происходит переход к другому аттрактору (бризеру другой конфигурации). Вопрос об устойчивости бризеров к внешним воздействиям является открытым и представляет собой предмет отдельного исследования.

\section{Финансирование работы}

А.П. Четвериков (разработка математической модели, описание структуры бассейнов притяжения) выражает благодарность за финансовую поддержку Российскому фонду фундаментальных исследований в рамках совместного с DFG научного проекта № 20-52-12004. К.С. Сергеев (проведение численного эксперимента, работа над текстом статьи) благодарит за финансовую поддержку Российский научный фонд в рамках проекта № 20-12-00119.

\section{Конфликт интересов}

Авторы заявляют, что у них нет конфликта интересов.

\section{Список литературы}

[1] Sievers A.J., Takeno S. // Phys. Rev. Lett. 1988. V. 61. P. 970 973.

[2] Дмитриев С.В., Корзникова Е.А., Баимова Ю.А., Веларде М.Г. // УФН. 2016. Т. 186. № 7. С. 471-488.

[3] Romanczuk P., Bär M., Ebeling W., Lindner B., SchimanskyGeier L. // Eur. Phys. J. Spec. Top. 2012. V. 202. P. 1-162.

[4] del Río E., Makarov V.A., Velarde M.G., Ebeling W. // Phys. Rev. E. 2003. V. 67. P. 056208.

[5] Thomson S.J., Couchman M.M.P., Bush J.W.M. arXiv:2001.09165v1 [pcond-mat.soft] 24 Jan 2020

[6] Endo T., Mori S. // IEEE Trans. Circuits Syst. 1978. V. 25. P. 7-18.

[7] Rosenblum M., Pikovsky A. // Phys. Rev. Lett. 2004. V. 92. P. 114102.

[8] Шабунин А.В., Акопов А.А., Астахов В.В., Вадивасова T.E. // Изв. вузов. Прикладная нелинейная динамика. 2005. T. 13. № 4. C. 37-55.

[9] Abrams D.M., Strogatz S.H. // Phys. Rev. Lett. 2004. V. 93. P. 174102.

[10] Chetverikov A.P., Sergeev K.S., del Rio E. // Int. J. Bifurcat. Chaos. 2018. V. 28. P. 1830027.

[11] Chetverikov A.P., Sergeev K.S., del Rio E. // Physica A. 2018. V. 513. P. $147-155$.

[12] Сергеев К.С., Четвериков А.П. // Нелинейная динамика. 2016. T. 12. № 3. C. 341-353. 